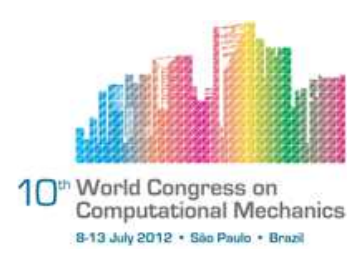

\title{
A DETAILED COMPARISON OF WENO AND SFV HIGH-ORDER METHODS FOR INVISCID AERODYNAMIC APPLICATIONS
}

\author{
L. G. L. Paula ${ }^{1}$, C. Breviglieri ${ }^{1}$, W. R. Wolf ${ }^{2}$, E. Basso ${ }^{2}$ and J. L. F. Azevedo ${ }^{3}$ \\ ${ }^{1}$ Instituto Tecnológico de Aeronáutica, DCTA/ITA \\ ${ }^{2}$ Instituto de Aeronáutica e Espaço, DCTA/IAE \\ ${ }^{3}$ Instituto de Aeronáutica e Espaço, DCTA/IAE (joaoluiz.azevedo@gmail.com)
}

\begin{abstract}
The purpose of this work is to compare two numerical formulations for unstructured grids that achieve high-order spatial discretization for compressible aerodynamic flows. High-order methods are necessary on the analysis of complex flows to reduce the number of mesh elements one would otherwise need if using traditional second-order schemes. In the present work, the 2-D Euler equations are solved numerically in a finite volume, cell centered context. The third-order Weighted Essentially Non-Oscillatory (WENO) and Spectral Finite Volume (SFV) methods are considered in this study for the spatial discretization of the governing equations. Time integration uses explicit, Runge-Kutta type schemes. Two literature test cases, one steady and one unsteady, are considered to assess the resolution capabilities and performance of the two spatial discretization methods. Both methods are suitable for the aerospace applications of interest. However, each method has characteristics that excel over the other scheme. The present results are valuable as a form of providing guidelines for future developments regarding high-order methods for unstructured meshes.
\end{abstract}

Keywords: High-order methods, Euler equations, WENO, Spectral Finite Volume.

\section{INTRODUCTION}

High-order reconstruction methods capable of handling unstructured grids are highly sought in computational fluid dynamics (CFD) since solving several real world problems require mesh discretizations over complex geometries. Examples of applications of such methods include computational aeroacoustics, large eddy simulation (LES), direct numerical simulation (DNS) and computational electromagnetics (CEM). High-order methods can be distinguished in formulation as structured or unstructured mesh support, and continuous or discontinuous reconstruction. State-of-the-art methods for unstructured, discontinuous highorder schemes include the Essentially Non-Oscillatory (ENO) and Weighted Essentially NonOscillatory (WENO) methods, Discontinuous Galerkin (DG), Spectral Finite Volume (SFV), Spectral Difference (SD) and Correction Procedure via Reconstruction (CPR) methods. In 
recent years, several efforts have been made by the CFD group of Instituto de Aeronautica e Espaco (DCTA/IAE) for the development of high-order unstructured grid schemes, such as the ENO, WENO and SFV methods, for the solution of aerodynamic flows with shock waves and contact surfaces [1,2]. Solution discontinuities pose a major challenge for research on highorder methods since reconstruction requires special treatment to preserve high-order accuracy and high resolution.

The numerical solver is currently implemented for the solution of the 2-D Euler equations in a cell centered finite volume context for unstructured meshes, with a Runge-Kutta scheme for time integration. The CFD tools developed in the group and used in the present work have been verified and validated previously [2, 3, 4, 5]. Furthermore, detailed analyses of the actual order of accuracy for the various schemes can also be found in the cited references. The paper, as here organized, presents the theoretical formulation of the spatial and temporal discretization methods for the Euler equations. The reconstruction process of the high-order polynomial for the SFV and WENO method is described next. Afterwards, a benchmark comparison of SFV and WENO formulations for two literature test cases are presented. These test cases consider the unsteady supersonic internal flow known as the forward-facing step problem [6] and the steady cold-gas flow through a hypersonic inlet [7]. A comparative analysis of the results for such cases is also presented with the objective of devising guidelines for future developments regarding high-order methods for unstructured meshes.

\section{THEORETICAL FORMULATION}

\subsection{Governing Equations}

In the present work, the 2-D Euler equations are solved in their integral form as

$$
\frac{\partial}{\partial t} \int_{V} Q d V+\int_{V}(\nabla \cdot \vec{P}) d V=0
$$

where $\vec{P}=E \hat{\imath}+F \hat{\jmath}$. The application of the divergence theorem to Eq. (1) yields

$$
\frac{\partial}{\partial t} \int_{V} Q d V+\int_{S}(\vec{P} \cdot \vec{n}) d S=0 .
$$

The vector of conserved variables, $Q$, and the convective flux vectors, $E$ and $F$, are given by

$$
Q=\left\{\begin{array}{c}
\rho \\
\rho u \\
\rho v \\
e_{i}
\end{array}\right\}, \quad E=\left\{\begin{array}{c}
\rho u \\
\rho u^{2}+p \\
\rho u v \\
\left(e_{i}+p\right) u
\end{array}\right\}, F=\left\{\begin{array}{c}
\rho v \\
\rho u v \\
\rho v^{2}+p \\
\left(e_{i}+p\right) v
\end{array}\right\} .
$$

The system is closed by the equation of state for a perfect gas

$$
p=(\gamma-1)\left[e_{i}-\frac{1}{2} \rho\left(u^{2}+v^{2}\right)\right]
$$

where the ratio of specific heats, $\gamma$, is set as 1.4 for all computations in this work.

In the finite volume context, Eq. (2) can be rewritten for the $i$-th control volume as

$$
\frac{\partial Q_{i}}{\partial t}=-\frac{1}{V_{i}} \int_{S_{i}}(\vec{P} \cdot \vec{n}) d S,
$$


where $Q_{i}$ is the cell averaged value of $Q$ at time $t$ and $V_{i}$ is the volume, or area in 2-D, on the $i$-th control volume.

\subsection{Spatial Discretization}

The spatial discretization process determines a $k$-th order discrete approximation to the integral in the right-hand side of Eq. (5). In order to solve it numerically, the computational domain, $\Omega$, with proper initial and boundary conditions, is discretized into $N$ non-overlapping elements such that

$$
\Omega=\bigcup_{i=1}^{N} S_{i}
$$

One should observe that the elements could be composed by any type of polygon, given that it is possible to decompose its bounding edges into a finite number of line segments $\Gamma_{K}$, such that

$$
S_{i}=\bigcup \Gamma_{K}
$$

In the present paper, however, the authors assume that the computational mesh is always composed of triangular elements. The boundary integral in Eq. (5) can be further discretized into the convective operator form

$$
C\left(Q_{i}\right) \equiv \int_{S_{i}}(\vec{P} \cdot \vec{n}) d S=\sum_{r=1}^{K} \int_{A_{r}}(\vec{P} \cdot \vec{n}) d S
$$

where $K$ is the number of faces, or edges in 2-D, of $S_{i}$, and $A_{r}$ represents the $r-t h$ edge of the element. Given the fact that $\vec{n}$ is constant for each line segment, the integration on the right side of Eq. (8) can be performed numerically with a $k-t h$ order accurate Gaussian quadrature formula

$$
\int_{A_{r}}(\vec{P} \cdot \vec{n}) d S=\sum_{r=1}^{K} \sum_{q=1}^{J} w_{r q} \vec{P}\left(Q\left(x_{r q}, y_{r q}\right)\right) \cdot \vec{n}_{r} A_{r}+O\left(A_{r} h^{k}\right) .
$$

where $\left(x_{r q}, y_{r q}\right)$ and $w_{r q}$ are, respectively, the Gaussian points and the weights on the $r$-th edge of $S_{i}, J=$ integer $((k+1) / 2)$ is the number of quadrature points required on the $r-t h$ edge, and $h$ will be defined in the forthcoming discussion. For the second-order schemes, one Gaussian point is used in the integration. Given the coordinates of the end points of the element edge, $z_{1}$ and $z_{2}$, one can obtain the Gaussian point as the middle point of the segment connecting the two end points, $G_{1}=\frac{1}{2}\left(z_{1}+z_{2}\right)$. For this case, the weight is $w_{1}=1$. For the third and fourth order schemes, two Gaussian points are necessary along each line segment. Their values are given by

$$
G_{1}=\frac{\sqrt{3}+1}{2 \sqrt{3}} z_{1}+\left(1-\frac{\sqrt{3}+1}{2 \sqrt{3}}\right) z_{2} \quad \text { and } \quad G_{2}=\frac{\sqrt{3}+1}{2 \sqrt{3}} z_{2}+\left(1-\frac{\sqrt{3}+1}{2 \sqrt{3}}\right) z_{1},
$$

and the respective weights, $w_{1}$ and $w_{2}$, are set as $w_{1}=w_{2}=0.5$. Using the method described above, one can compute values of $Q_{i}$ for instant $t$ for each mesh element. From these averaged values, it is possible to reconstruct polynomials that represent the conserved variables, $\rho, \rho u$, 
$\rho v$ and $e_{i}$. Due to the discontinuity of the reconstructed values of the conserved variables over the element boundaries, one must use a numerical flux function to approximate the flux values on the cell boundaries.

The above procedures follow exactly the standard finite volume method. The cellaveraged conservative variables, $\bar{q}$, at time $t$, for $S_{i}$ is computed as

$$
\bar{q}_{i, j}(t)=\frac{1}{V_{i}} \int_{S_{i}} q(x, y, t) d x d y,
$$

where $V_{i}$ is the volume of $S_{i}$. Once the cell-averaged conservative variables are available for all mesh elements, a polynomial, $p_{i}(x, y) \in P^{k-1}$, with degree $k-1$, can be reconstructed to approximate the $q(x, y)$ function inside $S_{i}$, i.e.,

$$
p_{i}(x, y)=q(x, y)+O\left(h^{k-1}\right), \quad(x, y) \in S_{i},
$$

where $h$ represents the maximum edge length of cell $S_{i}$. The polynomial reconstruction process is discussed in details in the following section and is the key difference between the WENO and SFV methods. For now, it is enough to say that this high-order reconstruction is used to update the cell-averaged state variables at the next time step for all the cells within the computational domain. Note that this polynomial approximation is valid within $S_{i}$ and some numerical flux coupling is necessary across element boundaries.

Integrating Eq. (5) in $S_{i}$, one can obtain the integral form for the averaged mean state variable

$$
\frac{d \bar{q}_{i}}{d t}+\frac{1}{V_{i}} \sum_{r=1}^{K} \int_{A_{r}}(f \cdot \vec{n}) d S=0,
$$

where $f$ represents the $E$ and $F$ fluxes, $K$ is the number of edges of $S_{i}$ and $A_{r}$ represents the $r-t h$ edge of the cell.

As previously stated, the flux integration across cell boundaries involves two discontinuous states, to the left and to the right of the edge. This flux computation can be carried out using an exact or approximate Riemann solver, or even a flux splitting procedure, which can be written in the form

$$
f\left(q\left(x_{r q}, y_{r q}\right)\right) \cdot \vec{n}_{r} \approx f_{\text {Riemann }}\left(q_{L}\left(x_{r q}, y_{r q}\right), q_{R}\left(x_{r q}, y_{r q}\right), \vec{n}_{r}\right),
$$

where $q_{L}$ is the conservative variable vector obtained by the $p_{i}$ polynomial applied at the $\left(x_{r q}, y_{r q}\right)$ coordinates and $q_{R}$ is the same vector obtained with the $p_{n b}$ polynomial in the same coordinates of the edge. Note that the $n b$ subscript represents the element to the right of the edge, whereas the $i$ subscript denotes the element to its left. As the numerical flux integration in the present paper is based on one of the forms of a Riemann solver, this is the mechanism which introduces the upwind and artificial dissipation effects into the methods, rendering them stable and accurate. In this work, the authors have used the Roe flux difference splitting method [8] to compute the numerical flux, i.e.,

$$
f_{\text {Riemann }}=f_{\text {roe }}\left(q_{L}, q_{R}, \vec{n}\right)=\frac{1}{2}\left[f\left(q_{L}\right)+f\left(q_{R}\right)-|\bar{B}|\left(q_{R}-q_{L}\right)\right],
$$

where $|\bar{B}|$ is Roe's dissipation matrix computed in the direction normal to the edges as

$$
|\bar{B}|=R|\bar{\Lambda}| R^{-1}
$$


Here, $|\bar{\Lambda}|$ is the diagonal matrix composed of the absolute values of the eigenvalues of the Jacobian matrix, evaluated using the Roe averages.

Finally, one ends up with the semi-discrete scheme for updating the control volumes, which can be written as

$$
\frac{d \bar{q}_{i}}{d t}=-\frac{1}{V_{i}} \sum_{r=1}^{K} \sum_{q=1}^{J} w_{r q} f_{\text {Riemann }}\left(q_{L}\left(x_{r q}, y_{r q}\right), q_{R}\left(x_{r q}, y_{r q}\right), \vec{n}_{r}\right) A_{r} .
$$

where the right hand side of Eq. (17) is the equivalent convective operator, $C\left(q_{i}\right)$, for the $i$-th control volume of the mesh.

\subsection{Temporal Discretization}

The temporal discretization is concerned with solving a system of ordinary differential equations. In the present work, the authors use a third-order, TVD Runge-Kutta scheme [9]. Rewriting Eq. (17) in a concise ODE form, one obtains

$$
\frac{d \bar{q}}{d t}=-\frac{1}{V_{i}} C(q)
$$

where

$$
\bar{q}=\left\{\begin{array}{c}
\bar{q}_{1} \\
\cdots \\
\bar{q}_{i} \\
\cdots \\
\bar{q}_{N}
\end{array}\right\}, \quad C(q)=\left\{\begin{array}{c}
C\left(q_{1}\right) \\
\cdots \\
C\left(q_{i}\right) \\
\cdots \\
C\left(q_{N}\right)
\end{array}\right\},
$$

and

$$
C\left(q_{i}\right)=-\frac{1}{V_{i}} \sum_{r=1}^{K} \sum_{q=1}^{J} w_{r q} f_{R o e}\left(q_{L}\left(x_{r q}, y_{r q}\right), q_{R}\left(x_{r q}, y_{r q}\right), \vec{n}_{r}\right) A_{r} .
$$

Hence, the time marching scheme can be written as

$$
\begin{aligned}
\bar{q}^{(1)} & =\bar{q}^{n}+\Delta t C\left(q^{n}\right), \\
\bar{q}^{(2)} & =\alpha_{1} \bar{q}^{n}+\alpha_{2}\left[\bar{q}^{(1)}+\Delta t C\left(q^{(1)}\right)\right], \\
\bar{q}^{(n+1)} & =\alpha_{3} \bar{q}^{n}+\alpha_{4}\left[\bar{q}^{(2)}+\Delta t C\left(q^{(2)}\right)\right],
\end{aligned}
$$

where the $n$ and $n+1$ superscripts denote, respectively, the values of the properties at the beginning and at the end of the $n$-th time step. The $\alpha$ coefficients are $\alpha_{1}=3 / 4, \alpha_{2}=1 / 4$, $\alpha_{3}=1 / 3$ and $\alpha_{4}=2 / 3$.

\section{SPECTRAL FINITE VOLUME RECONSTRUCTION}

\subsection{General Formulation}

The evaluation of the conserved variables at the quadrature points is necessary in order to perform the flux integration over the mesh element edges. These evaluations can be achieved by reconstructing conserved variables in terms of some base functions using the DOFs within a mesh element, denoted here as a Spectral Volume, or SV for short [10, 11]. 
The present work has carried out such reconstructions using polynomial functions. Let $P_{m}$ denote the space of $m$-th degree polynomials in two dimensions. Then, the minimum dimension of the approximation space that allows $P_{m}$ to be complete is

$$
N_{m}=\frac{(m+1)(m+2)}{2} \text {. }
$$

In order to reconstruct $q$ in $P_{m}$, it is necessary to partition the $\mathrm{SV}$ into $N_{m}$ non-overlapping control volumes, or CVs, such that

$$
S_{i}=\bigcup_{j=1}^{N_{m}} C_{i, j} .
$$

The reconstruction problem, for a given continuous function in $S_{i}$ and a suitable partition, can be stated as finding $p_{m} \in P_{m}$ such that

$$
\int_{C_{i, j}} p_{m}(x, y) d S=\int_{C_{i, j}} q(x, y) d S .
$$

With a complete polynomial basis, $e_{l}(x, y) \in P_{m}$, it is possible to satisfy Eq. (23). Hence, $p_{m}$ can be expressed as

$$
p_{m}=\sum_{l=1}^{N_{m}} b_{l} e_{l}(x, y)
$$

where $e$ is the base function vector, $\left[e_{1}, \cdots, e_{N}\right]$, and $b$ is the reconstruction coefficient vector, $\left[b_{1}, \cdots, b_{N}\right]^{T}$. The substitution of Eq. (24) into Eq. (23) yields

$$
\frac{1}{V_{i, j}} \sum_{l=1}^{N_{m}} b_{l} \int_{C_{i, j}} e_{l}(x, y) d S=\bar{q}_{i, j} .
$$

If $\bar{q}$ denotes the $\left[\bar{q}_{i, 1}, \cdots, \bar{q}_{i, N m}\right]^{T}$ column vector, Eq. (25) can be rewritten in matrix form as

$$
S b=\bar{q},
$$

where the $S$ reconstruction matrix is given by

$$
S=\left[\begin{array}{ccc}
\frac{1}{V_{i, 1}} \int_{C_{i, 1}} e_{1}(x, y) d S & \cdots & \frac{1}{V_{i, 1}} \int_{C_{i, 1}} e_{N}(x, y) d S \\
\vdots & \cdots & \vdots \\
\frac{1}{V_{i, N}} \int_{C_{i, N}} e_{1}(x, y) d S & \cdots & \frac{1}{V_{i, N}} \int_{C_{i, N}} e_{N}(x, y) d S
\end{array}\right]
$$

and, then, the reconstruction coefficients $b$ can be obtained as

$$
b=S^{-1} \bar{q},
$$

provided that $S$ is non-singular. With the substitution of Eq. (28) into Eq. (23), $p_{m}$ is, then, expressed in terms of shape functions $L=\left[L_{1}, \cdots, L_{N}\right]$, defined as $L=e S^{-1}$, such that one could write

$$
p_{m}=\sum_{j=1}^{N_{m}} L_{j}(x, y) \bar{q}_{i, j}=L \bar{q} .
$$


Table 1. Polynomial base functions.

\begin{tabular}{cc}
\hline Reconstruction Order & $e$ \\
\hline linear & {$[1 x y]$} \\
quadratic & {$\left[1 x y x^{2} x y y^{2}\right]$} \\
cubic & {$\left[1 x y x^{2} x y y^{2} x^{3} x^{2} y x y^{2} y^{3}\right]$} \\
\hline
\end{tabular}

Equation (29) gives the value of the conserved state variable, $q$, at any point within the SV and its boundaries, including the quadrature points, $\left(x_{r q}, y_{r q}\right)$.

The major advantage of the SFV method is that the reconstruction process does not need to be carried out for every mesh element $S_{i}$. One can compute these coefficients as a pre-processing step and they do not change along the simulation. This is a major difference when compared to methods such as ENO and WENO, for which every mesh element has a different reconstruction process at each time step. The polynomial base functions for the linear, quadratic and cubic reconstructions are listed in Table 1. Clearly, the linear, quadratic and cubic polynomial reconstructions will yield, respectively, 2nd-, 3rd- and 4th-order spatial discretization numerical schemes.

\subsection{Linear Reconstruction}

For the linear SFV method reconstruction, $m=1$, one needs to partition a SV in three sub-elements as in Eq. (21) and use the base vector as defined in Table 1. The partition scheme is given for a standard element. The partition for this case is uniquely defined. The structured aspect of the CVs within the SVs is used to determine neighborhood information and generate the global connectivity data considering a hash table search algorithm [12].

The linear partition is presented in Fig. 1(a). It yields a total of 7 points, 9 edges ( 6 are external edges and 3 are internal ones) and 9 quadrature points. The linear polynomial for the SFV method depends only on the base functions and on the partition shape. The integrals of the reconstruction matrix in Eq. (27) are obtained analytically [13] for fundamental shapes. The shape functions, in the sense of Eq. (29), are calculated and stored in memory for the quadrature points, $\left(x_{r q}, y_{r q}\right)$, of the standard element. Such shape functions have the exact same value for the quadratures points of any other SV of the mesh, provided they all have the same partition. There is one quadrature point located at the middle of the every CV edge.

\subsection{Quadratic Reconstruction}

For the quadratic reconstruction, $m=2$, one needs to partition a SV in six subelements and use the base vector as defined in Table 1. The partition scheme is also given in this work for a right triangle. The nodes of the partition are obtained in terms of barycentric coordinates of the SV element nodes in the same manner as the linear partition. The structured aspect of the CVs within the SVs is used to determine neighborhood information and generate the connectivity table. The ghost creation process and edge-based data structure is the same as for the linear reconstruction case. The partition used in this work [14] is shown in Fig. 1(b). It has a total of 13 points, 18 edges (9 external edges and 9 internal ones) and 36 


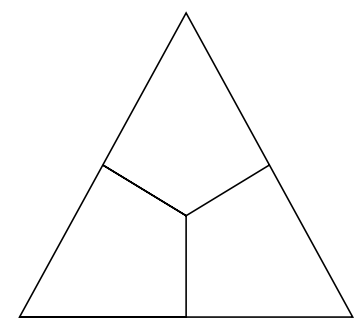

(a)

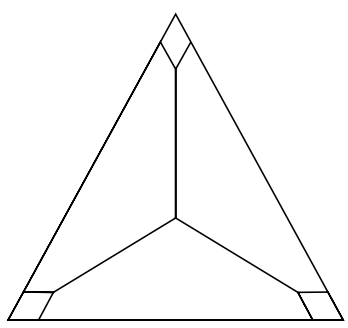

(b)

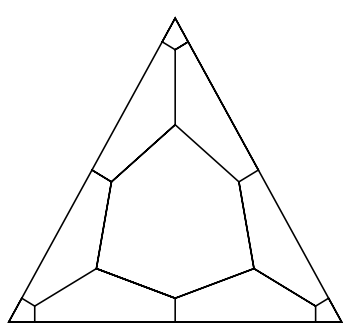

(c)

Figure 1. Triangular spectral volume partitions for (a) linear, (b) quadratic and (c) cubic reconstructions.

quadrature points. The shape functions, in the sense of Eq. (29), are obtained as in the linear partition. The reader should note that, in this case, the base functions have six terms that shall be integrated. Again, these terms are obtained exactly and kept in memory [13]. In this case, two quadrature points are required per CV edge.

\subsection{Cubic Reconstruction}

For the cubic reconstruction, $m=3$, one needs to partition the SV in ten sub-elements and to use the base vector as defined in Table 1. The ghost creation process and edge-based data structure is the same as for the linear and quadratic reconstruction cases. As a matter of fact, the same algorithm utilized to perform these tasks can be applied to higher order reconstructions. The partition used in this work is the improved cubic partition [14], presented in Fig. 1(c) and it has a total of 21 points, 30 edges (12 external edges and 18 internal ones) and 60 quadrature points. The shape functions, in the sense of Eq. (29), are obtained as in the linear partition in a pre-processing step. As with the quadratic reconstruction, each CV edge has two quadrature points [4].

\section{WENO RECONSTRUCTION}

\subsection{General Formulation}

The reconstruction procedure of the ENO schemes is based on the approximation of mean values of the primitive variables for each cell in the mesh by polynomials of one order less than the spatial order of accuracy expected. For the construction of polynomials of $\eta$-th order, one must use $N_{m}$ ) cells, where $N_{m}$ is defined as for the SFV method in Eq. (21). The first step in obtaining the polynomial reconstruction for each cell is to define the possible set of cells, called a stencil, that will be used. In the finite volume cell centered scheme, the stencils can be selected in a von Neumann neighborhood for a linear polynomial reconstruction (second-order accuracy). This approach can be extended to higher orders through the use of von Neumann neighborhoods of the primary neighbors already selected for the second-order reconstruction. In the present work, the cells are triangles and the $p(x, y)$ polynomials can be 
calculated as

$$
p(x, y)=\sum_{|\beta| \leq \eta} r_{\beta_{1} \beta_{2}}\left(x-x_{c}\right)^{\beta_{1}}\left(y-y_{c}\right)^{\beta_{2}},
$$

where $|\beta|=\beta_{1}+\beta_{2}$, with $\beta_{i} \in\{0,1,2, \ldots\}, x_{c}$ and $y_{c}$ are the Cartesian coordinates of the barycenter of the control volumes and $r_{\beta_{1} \beta_{2}}$ are unknown coefficients which are some approximations to the derivatives of the primitive variables.

Once it is established that $p(x, y)$ is a good approximation to the mean values of primitive variables for each cell, one can write a linear system, $[R]\{r\}=\{\bar{u}\}$, of $N(\eta)$ equations for the $N(\eta)$ unknowns, $r_{\beta_{1} \beta_{2}}$. Here, $[R]$ is the matrix of control volume moments, as in Gooch [15], computed using the scaling technique proposed by Friedrich [16] to circumvent a poorly conditioned matrix. Moreover, $\{r\}$ is the vector of unknown coefficients that must be found and $\{\bar{u}\}$ is the vector composed by the mean values for each primitive variable. The stencil is considered admissible if the $[R]$ matrix is invertible. The control volume moments that compose the $[R]$ matrix are defined by Eq. (31) as

$$
\overline{x^{\varphi} y^{\zeta}} \equiv \frac{1}{V_{i}} \int_{S_{i}}\left(x-x_{c}\right)^{\varphi}\left(y-y_{c}\right)^{\zeta} d S,
$$

and are evaluated using Gauss quadrature formulae following the same procedure as the one used in the flux computation. After the polynomial reconstruction is performed for each cell, the next step is to verify which polynomial is the least oscillatory to use in the ENO scheme. The oscillation is computed using some indicator that assesses the smoothness of $p(x, y)$.

Following the results presented in the literature [16, 3], the oscillation indicator used in the present work is the one proposed by Jiang and Shu [17], which was later modified by Friedrich [16]. The formulation for this oscillation indicator can be expressed as

$$
O I_{J S}(p(x, y))=\left[\sum_{1 \leq|\beta| \leq \eta} \int_{V_{i}} h^{2|\beta|-4}\left(\frac{\partial^{|\beta|} p(x, y)}{\partial x^{\beta 1} \partial y^{\beta 2}}\right)^{2} d x d y\right]^{\frac{1}{2}},
$$

where $h$ is the mesh width.

Differently from the ENO schemes, the WENO schemes use all the calculated polynomials [3]. These polynomials are added together through the use of weights which are computed for each one of the polynomials as proportional to its respective oscillation indicator. The main idea in the WENO reconstruction is to attribute the computed weights for each polynomial with the aim of reconstructing a new polynomial as $p(x, y)=\sum_{k=1}^{m} \omega_{k} p_{k}(x, y)$. The weights are of order one in the smooth regions of the flow and are of the order of the desired accuracy in the solution in the regions with discontinuities. The weights can be computed as

$$
\omega_{k}=\frac{\left[\epsilon+O I\left(p_{k}(x, y)\right)\right]^{-\theta}}{\sum_{k^{\prime}=1}^{m}\left[\epsilon+O I\left(p_{k^{\prime}}(x, y)\right)\right]^{-\theta}},
$$

where $\epsilon$ is a small real number used to avoid division by zero and $\theta$ is a positive integer. The WENO schemes have the property of being very smooth and stable in smooth regions of the flow, but this property is lost if $\theta$ is chosen too large. In that case the scheme tends to behave like the ENO schemes. In the present work, the $\theta$ term is chosen as $\theta=2$ because this yielded the best convergence rates in the results. 


\subsection{Stencil Selection Algorithm}

In Refs. [1] and [3], reconstruction of second-order accurate ENO and WENO schemes was presented. Details regarding to stencil selection algorithms were discussed and results appearing in these cited references demonstrated the accuracy of the second-order accurate ENO and WENO methods. For the implementation of the third-order accurate schemes one must use six cells for the polynomial reconstructions. Five unknown coefficients are computed for each polynomial through the solution of a five equation linear system composed by the control volume moments and the primitive variables of the cells considered in the stencil. The algorithm implemented in this work is based on the ideas proposed by Abgrall [18]. The first step consists in finding the stencil that presents the smoothest oscillation among the possible stencils for a linear reconstruction using only the primary neighbors of the main control volume. This algorithm uses this concept for both the ENO and WENO schemes for meshes composed by triangles and quadrilaterals. After the smoothest three-cell stencil is chosen, one must start the procedure for the third-order accuracy reconstruction. If the control volume is a triangle one has a maximum of 4 secondary neighbors for the reconstruction and, therefore, 4 possibilities of stencils for the selection. However, if the control volume is a quadrilateral, one has a maximum of 6 secondary neighbors for the reconstruction and, therefore, 20 possibilities of stencils for the selection. In Fig. 2 one can see a typical neighborhood for the implementation of the algorithm with the hatched volumes representing a possible stencil for this algorithm.

Although the case where one has 20 stencils for the selection can occur, in most cases, one has 5 secondary neighbors for the reconstruction in quadrilaterals and, then, 10 possibilities of stencils for the selection. Such a situation happens because, when selecting the stencil with the primary neighbors, one has, in most cases, a stencil that is not centered as can be viewed in Fig. 3 for the control volumes labeled NG1 and NG2. In such case, one can see that there are 5 secondary neighbors (the control volumes labeled as $2 \mathrm{nd} \mathrm{NG).} \mathrm{In} \mathrm{triangular} \mathrm{control}$ volumes, one can have the presence of three secondary neighbors for the reconstruction, which is the worst case for reconstruction on a triangular mesh. A case such this can be observed in Fig. 4, where the hatched control volumes NG1 and NG2 compose the smoothest stencil for a second-order reconstruction and the hatched control volumes, NG3, NG4 and NG5, are the control volumes used for the third-order reconstruction. As one can see, this case allows the construction of only one stencil for the selection. If this stencil is in a discontinuous region of the flow, it will produce oscillations, and it will lead to a non-physical solution. Hence, the procedure adopted to avoid the problem where one has a stencil such this in a discontinuous region of the flow is to reduce the order of accuracy of the scheme in these cells.

As mentioned in the papers of Abgrall [18] and Harten and Chakravarthy [19], one has to reduce the order of accuracy in some cells to maintain the stability of the schemes for both the WENO schemes. The technique used for the reduction of the order of accuracy is the same presented by Abgrall [18]. Moreover, it should be pointed out that the WENO scheme can handle triangular and quadrilateral elements, including a mix of these, for a given mesh. However, for the sake of comparison with the SFV method, only triangular meshes are considered in this study. 

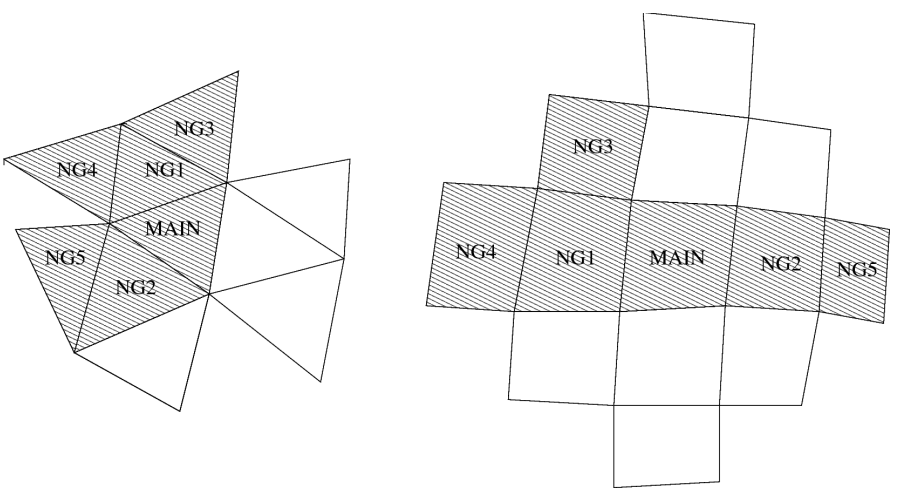

Figure 2. Typical neighborhood for a third-order accuracy reconstruction. The hatched volumes exemplify a possible stencil for the reconstruction.

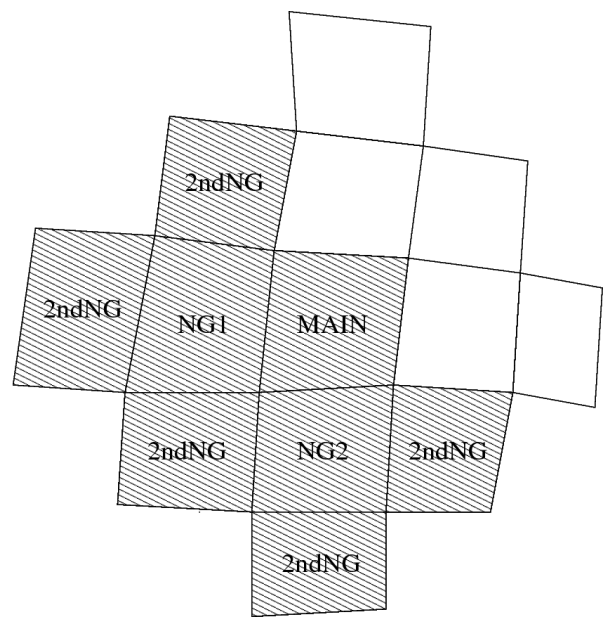

Figure 3. Neighborhood for a third-order accuracy reconstruction in a mesh composed exclusively by quadrilaterals. The 5 different hatched volumes marked as $2 \mathrm{ndNG}$ can compose only 10 stencils, instead of the 20 stencils that could be composed if the cells NG1 and NG2 were centered, as in Fig. 2.

\section{RESULTS}

The results presented here attempt to validate both the implementation of the data structure, temporal integration, numerical stability and resolution of the high-order methods. For the presented results, density is made dimensionless with respect to the free stream condition and pressure is made dimensionless with respect to the density times the speed of sound squared. Moreover, the $L_{\max }$ norm of the residue is used for the convergence history plots.

\subsection{Forward-Facing Step}

The forward step test case is designed to resolve complex oblique shock reflections, pertinent to supersonic variable-geometry jet engine intakes. Due to computational constraints faced by Emery [20], the test case was designed to be numerically simple to set up. The initial conditions are uniform throughout the domain, and the inlet boundary condition is supersonic. 


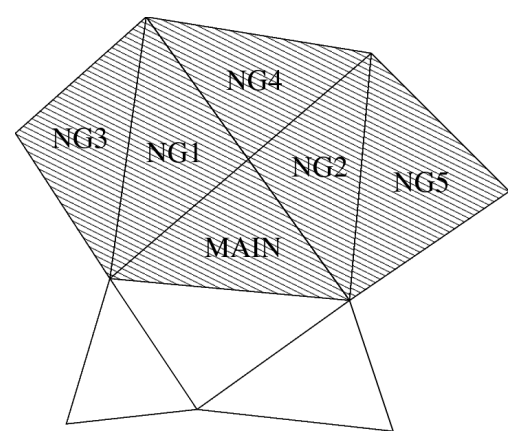

Figure 4. Possible stencil for a third-order accuracy reconstruction. The second-order stencil composed by the cells NG1 and NG2 was selected as the smoothest. Then, due to the lack of possibilities to switch the cells, one have to reduce the order of accuracy in cases where this stencil is in a discontinuous region of the flow.

Such setup is actually difficult to perform experimentally, due to the unrealistic combination of initial and boundary conditions. However, quantitative results are available for a range of numerical methods [6], which makes this a good case for validating the capabilities of the present flow solver independently of the numerical method used.

The artificial nature of the test setup helps to evaluate the robustness of the spatial discretization algorithm combined with the limiter technique. Due to the strong shock reflection at the lower face of the step during the first few iterations, it is difficult to maintain positivity of pressure and density using various numerical schemes. Furthermore, the edge of the forward step is a singular point of the Prandtl-Meyer expansion fan generated by the flow over the step. The continuity and momentum equations can disobey the second law of thermodynamics through an expansion fan. This introduces numerical difficulty in the form of a nonphysical expansion shock, which at high Mach numbers and small cell sizes can yield negative pressures and densities in the code.

The 2-D configuration is 3 dimensionless length units long and 1 unit wide, with a step of 0.2 units high located at 0.6 units from the configuration inlet. The inflow and outflow boundary conditions are both supersonic, so the solver does not have to account for waves leaving the domain at the entrance boundary, or entering the domain at the exit boundary. An uniform Mach number of 3.0 is set as inflow at $t=0$ dimensionless time units. One should observe that this test case is unsteady. The mesh has has 7022 nodes and 13608 triangular elements and is used for both schemes. The more interesting structure of the flow develops at time equal to $t=4.0$ dimensionless time units. Results at this instant of time appear in Figs. 5 and 6 , for the computation with 3rd-order WENO and SFV schemes.

At the instant of time presented in the solutions, a detached shock evolves to a lambda shock that reflects in the upper surface of the channel. A contact discontinuity is created past the lambda shock and both the reflected shock and the contact discontinuity move downstream along the channel. The reflected shock is again reflected at the lower wall as well as near the end of the channel. The contact discontinuity interacts with the shock that reflected in the lower wall. This interaction occurs in the region near the end of the channel, just upstream of the last reflection of the shock. At the corner region, there is also a weak oblique shock wave that ends the expansion region due to a Prandtl-Meyer expansion fan. This weak shock 


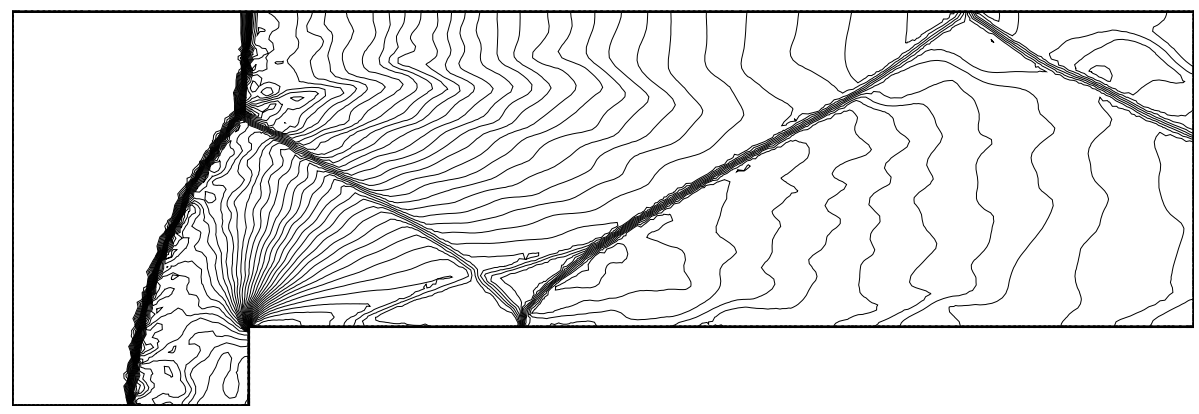

(a) Third-order WENO method solution.

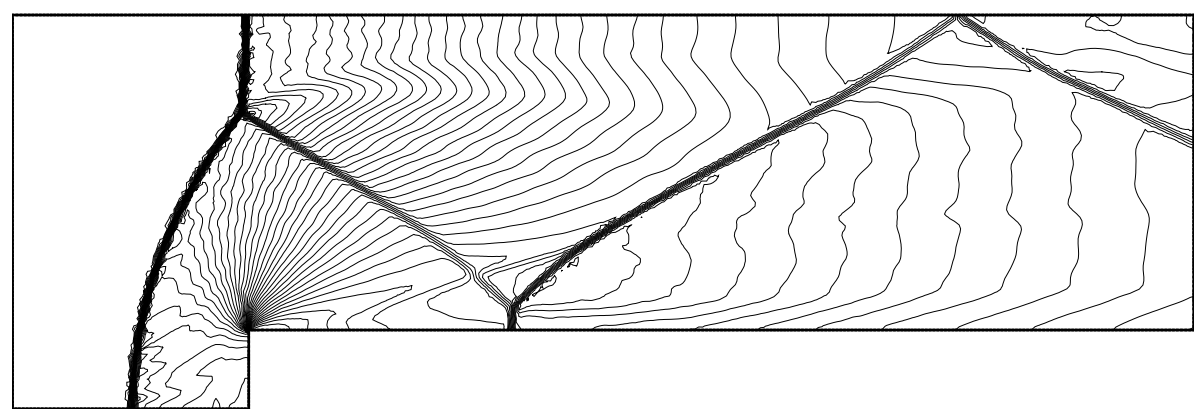

(b) Third-order SFV method solution.

Figure 5. Forty evenly-spaced dimensionless density contours lines from 0.05 to 4.62 at $t=$ 4.0 dimensionless time units.

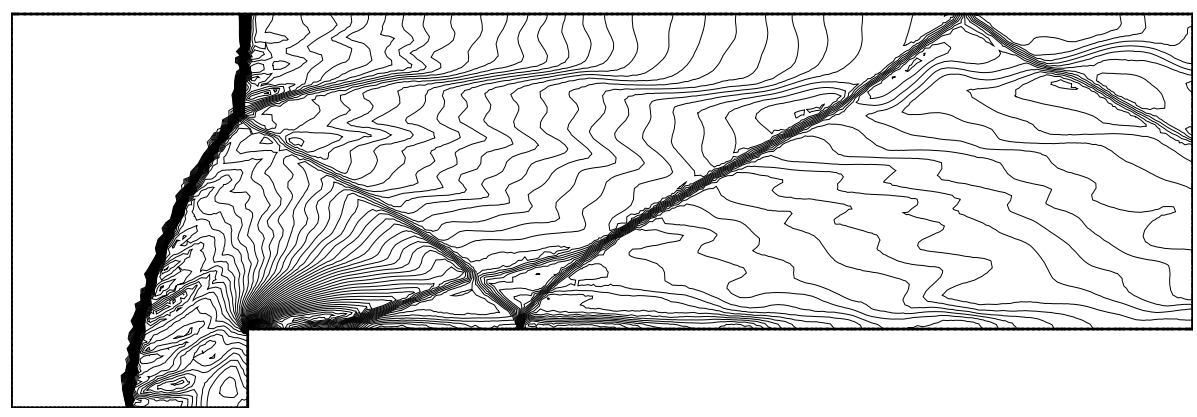

(a) Third-order WENO method solution.

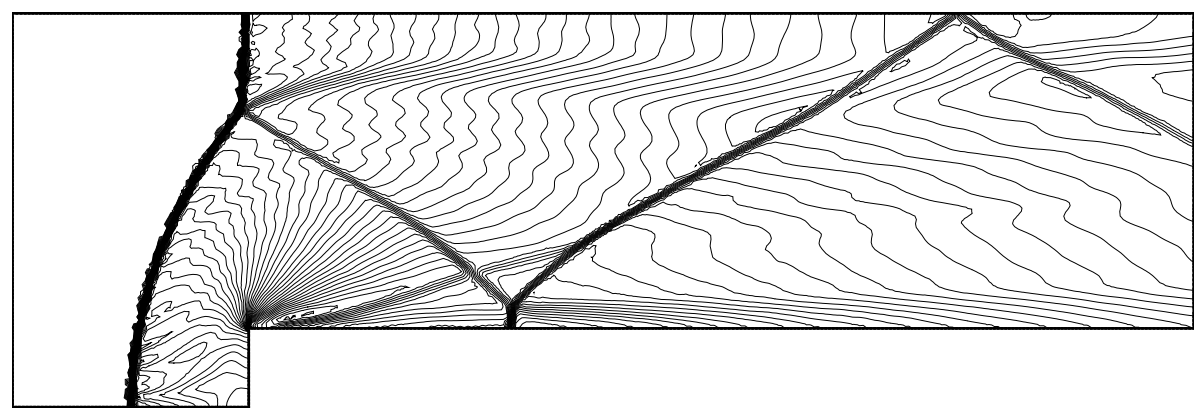

(b) Third-order SFV method solution.

Figure 6. Sixty evenly-spaced Mach number contour lines from 0.01 to 3.8 at $\mathrm{t}=4.0$ dimensionless time units. 
Table 2. Iteration cost estimates.

\begin{tabular}{ccc}
\hline \hline Method & Order & time \\
\hline SFV & 3 & 1.0 \\
WENO & 3 & 4.8 \\
\hline \hline
\end{tabular}

interacts with the first reflected shock near the lower wall of the channel. This leads typically to a flow structure that resembles an unphysical shock-boundary layer interaction in the region where the second shock reflection occurs. The solution in this region is very dependent on the treatment applied to the corner of the step. In the present work, no special treatment was applied to the corner of the step, contrary to the original reference [6]. One can see that the results here presented are similar to those presented in Refs. [18] and [21], where, as in this work, no special treatment was applied to the corner of the step. The use of a solution limiter formulation is required for the SFV method to preserve the positivity of the reconstruction. More details on the limiter formulation is given in Ref. [2].

The results obtained present a difference regarding the height where the lambda shock is positioned. One can see that the lambda shock forms closer to the wall for the third-order WENO scheme and this affects the position of the contact discontinuity. In Fig. 6, one can see that the WENO scheme presented the shock-boundary layer interaction created past the corner region in the lower wall of the channel, although such effect is much less pronounced in the SFV method solution plot. In other words, the second reflection of the shock presented a Y shock instead of a simple reflection in the wall. Both methods captured the weak shock near the corner region very well, as in the results shown in Sonar [21]. All the shock reflections have a better resolution for the third-order SFV scheme and they are in the correct positions compared to Ref. [6]. The overall simulation cost is presented in Table 2, where the relative iteration costs are normalized by the 3rd-order SFV scheme.

\subsection{Hypersonic Inlet}

The second test case discusses the computational results of the cold gas hypersonic flow in a 2-D inlet configuration which is representative of some proposed inlet geometries for typical trans-atmospheric vehicles. This test case is well documented in Ref. [7]. For the present simulations, the fluid is treated as a perfect gas with constant specific heat and no chemistry is taken into account. The purpose of the simulations is to compare the solutions of the implemented high-order schemes in high Mach number flow conditions. Such simulations are aimed at verifying if these schemes are able to represent all flow features, such as strong shocks, shock reflections and interactions, and expansion regions. Moreover, there is interest in verifying whether the schemes can avoid oscillations in the presence of such strong discontinuities. The results considering an inlet entrance Mach number $M_{e}=4$ are discussed in the present work.

The comparison among the third-order WENO and SFV method results is presented in Figs. 7 and 8. The simulations are performed on a mesh with 2691 nodes and 5000 triangles. The reader can observe in Fig. 8 the strong oblique shocks formed along the walls of the inlet 


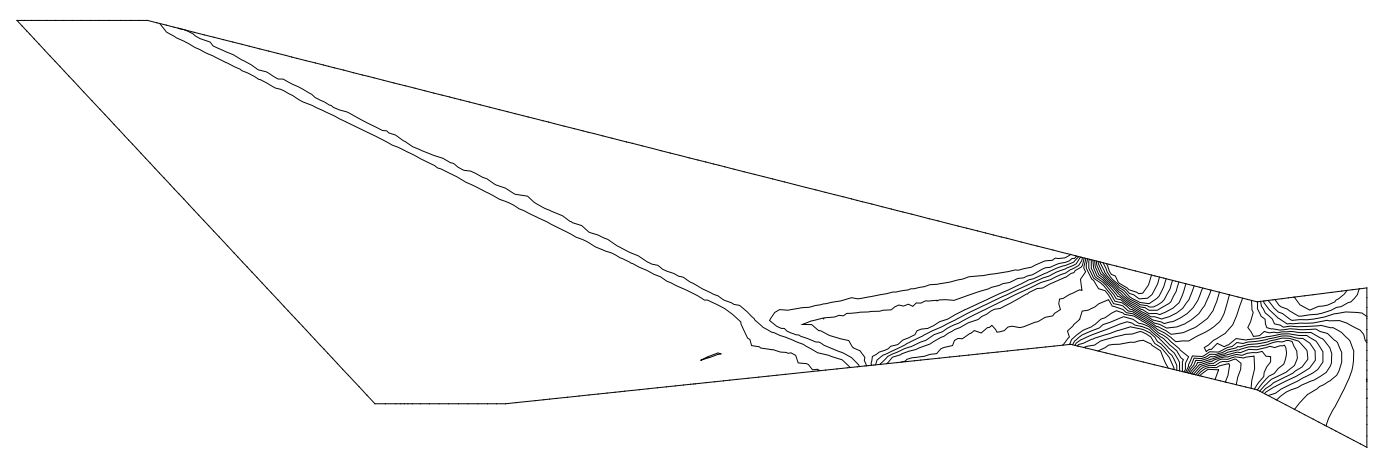

(a) Third-order WENO method solution.

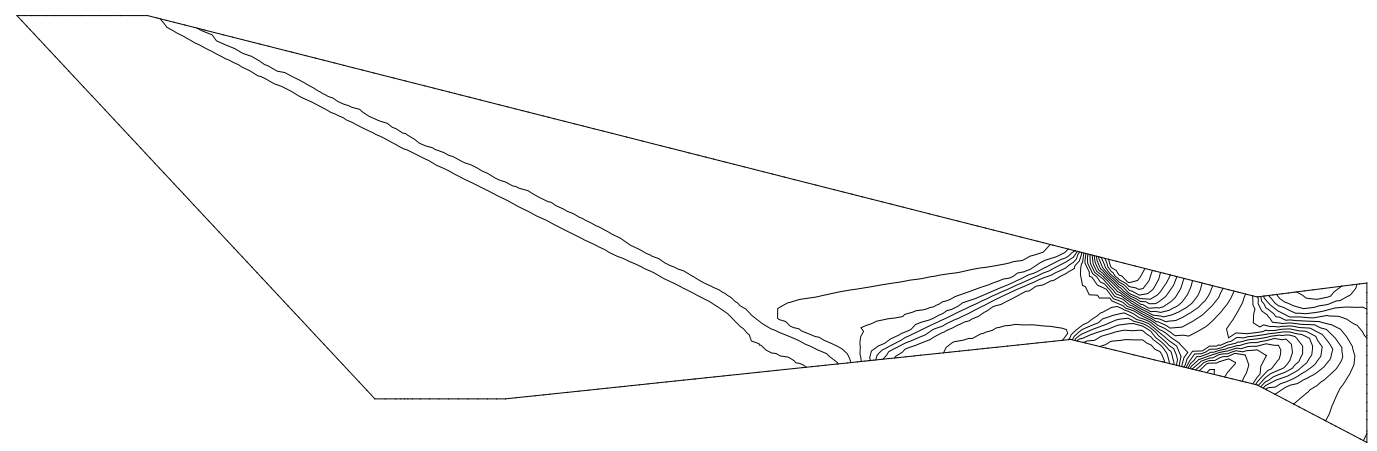

(b) Third-order SFV method solution.

Figure 7. Twenty evenly-spaced dimensionless density contour lines from 1.5 to 12.

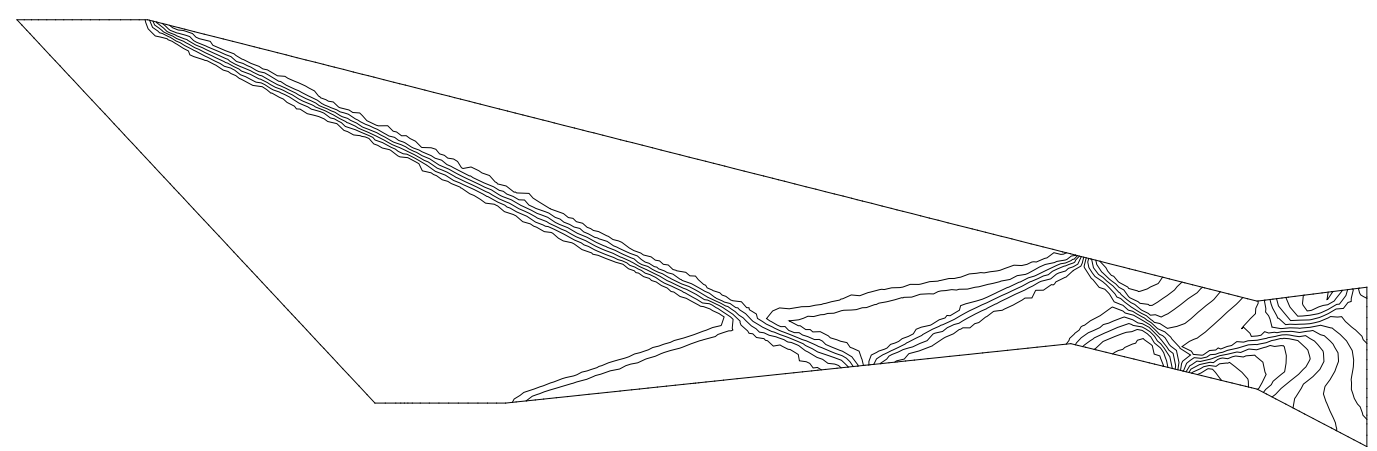

(a) Third-order WENO method solution.

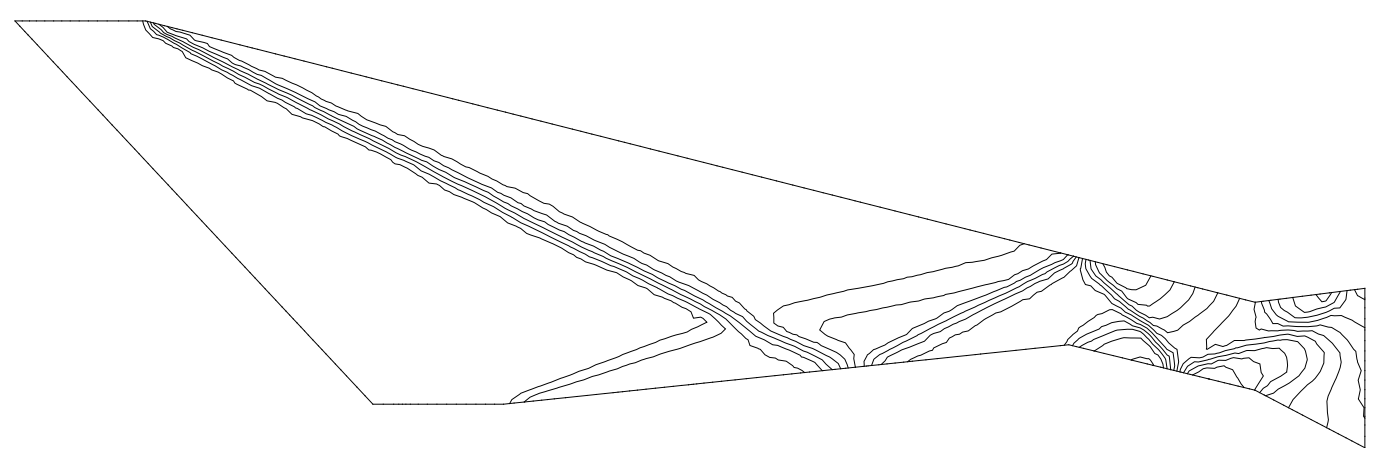

(b) Third-order SFV method solution.

Figure 8. Forty evenly-spaced Mach number contour lines from 1.0 to 4.0. 
entrance. Such shock waves produce shock-shock interactions as well as an expansion fan in the lower wall that interacts with a shock wave resulting from the shock-shock interaction. The shock wave which interacts with the expansion fan reflects in the lower wall of the inlet, and the other shock wave resulting from the shock-shock interaction reflects in the upper wall. Downstream of this region, one can observe an expansion fan formed in the upper wall corner and another expansion fan in the second lower wall corner. Furthermore, another shock-shock interaction occurs near the exit region of the inlet. Finally, there is a last shock reflection in the lower wall, near the exit region of the inlet. One can observe that the physical characteristics of the flow are better captured for the simulation performed with the WENO scheme. Since the SFV method employes a limiter formulation, to render the simulation stable, it presents a tendency to smooth out the discontinuities.

Figure 9 presents the residue history for both high-order schemes. The residues are normalized by their first iteration value. The reader should note that this test case presents a steady-state solution. Moreover a comparison between the methods performance show a critical aspect of the WENO method regarding numerical convergence. Since the adaptive stencil procedure is embebbed within the WENO scheme formulation, a steady or fixed set of stencil for polynomial reconstruction is hard to achieve. This translates into a difficulty for convergence to machine zero for steady solutions. On the other hand, the SFV method uses a fixed set of cells to perform the polynomial reconstruction that do not depend on the solution itself. Such aspect of the SFV method enables the convergence to machine-zero as indicated on the figure.

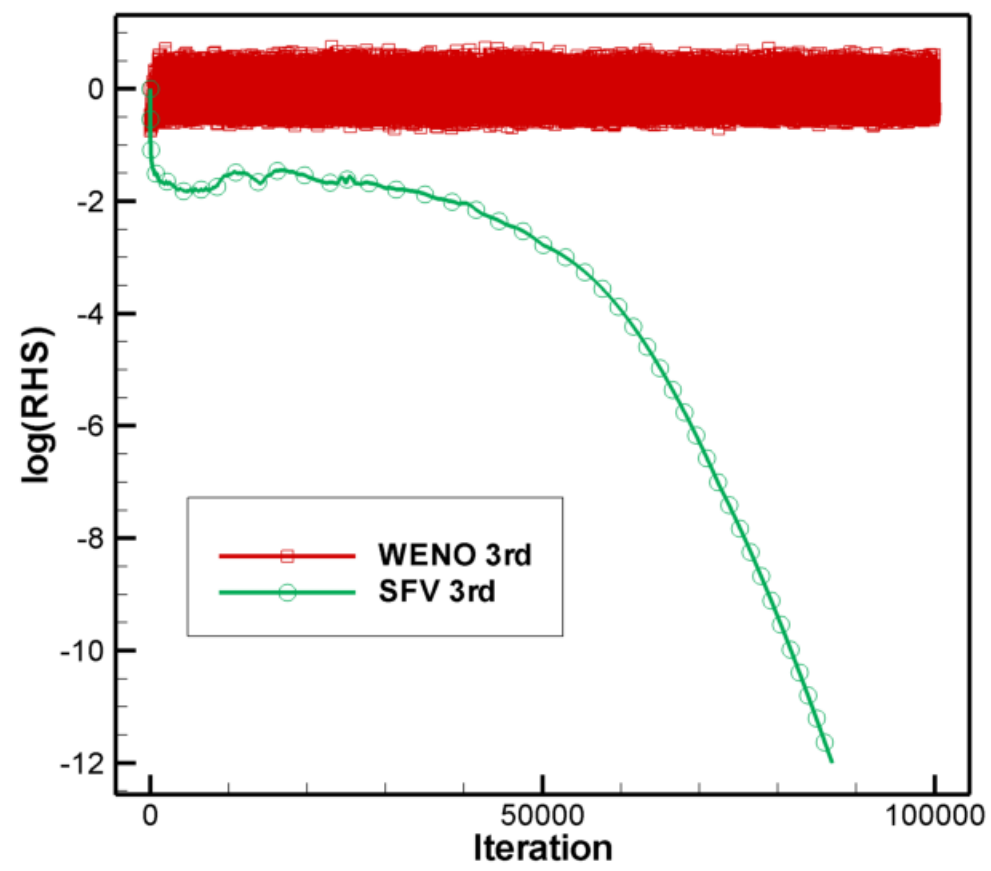

Figure $9 . L_{\infty}$-norm for density residue. 
Table 3. Iteration cost estimates.

\begin{tabular}{ccc}
\hline \hline Method & Order & time \\
\hline SFV & 3 & 1.0 \\
WENO & 3 & 1.9 \\
\hline \hline
\end{tabular}

The overall simulation cost is presented in Table 3, where the relative iteration costs are normalized by the 3rd-order SFV scheme. It is important to note that the SFV method cost is increased for the results here presented due to the limiter formulation, as described in Ref. [2]. Even though, the SFV method outperforms the WENO method for the same order of accuracy.

\section{CONCLUSIONS}

The SFV and WENO high-order method formulations are presented and compared against well-known literature test cases. The resolution of the methods is shown to be in good agreement with each other. Furthermore, the SFV method tend to smooth out some flow features such as the shock waves, due to the limiter necessary for solution stability. The WENO method, on the other hand, does not require an explicit limiter formulation and it clearly shows better resolved flow features. However, from a computational efficiency standpoint, the SFV method outperforms the same order WENO method. The cost per iteration and residue convergence rate are in favor of the SFV method. This should be expected due to the fixed cell stencil for polynomial reconstruction used by the SFV scheme.

Both methods are suitable for the aerospace applications of interest, to the computational aerodynamics laboratory at IAE. At the present moment, however, each method has characteristics that excel over the other scheme. The WENO search algorithms, for instance, represent a real challenge for parallel applications but requires no limiter technique for dealing with the strong shocks that appear in supersonic and hypersonic flows. The SFV method, on the other hand, can be easily implemented in a parallel framework, but requires order reduction by a limiter formulation to be stable for the same applications. The present results are valuable to aid researchers choose the appropriate method for the problem at hand and also provide guidelines for future developments regarding high-order methods for unstructured meshes.

\section{Acknowledgements}

The authors gratefully acknowledge the partial support for this research provided by Conselho Nacional de Desenvolvimento Científico e Tecnológico, CNPq, under the Research Grants No. 312064/2006-3 and No. 471592/2011-0. This work is also supported by Fundação Coordenação de Aperfeiçoamento de Pessoal de Nível Superior, CAPES, through a Ph.D. Scholarship for the second author. The authors are also indebted to the financial support received from FAPESP under Grant No. 2011/12493-6. 


\section{REFERENCES}

[1] Wolf, W. R., and Azevedo, J. L. F., "High-Order ENO and WENO Schemes for Unstructured Grids," International Journal for Numerical Methods in Fluids, Vol. 55, No. 10, Dec. 2007, pp. 917-943.

[2] Breviglieri, C., Azevedo, J. L. F., Basso, E., and Souza, M. A. F., "Implicit High-Order Spectral Finite Volume Method for Inviscid Compressible Flows," AIAA Journal, Vol. 48, No. 10, Oct. 2010, pp. 2365-2376.

[3] Wolf, W. R., and Azevedo, J. L. F., "High-Order Unstructured Essentially Nonoscillatory and Weighted Essentially Nonoscillatory Schemes for Aerodynamic Flows," AIAA Journal, Vol. 44, No. 10, Oct. 2006, pp. 2295-2310.

[4] Breviglieri, C., Basso, E., and Azevedo, J. L. F., "High-Order Unstructured Spectral Finite Volume Scheme for Aerodynamic Applications," AIAA Paper No. 2008-7182, 26th AIAA Applied Aerodynamics Conference, Honolulu, HI, Aug. 2008.

[5] Breviglieri, C., and Azevedo, J. L. F., "Unsteady Aerodynamic Applications Using High-Order Unstructured Grid Methods," AIAA Paper No. 2012-0701, 50th AIAA Aerospace Sciences Meeting Including the New Horizons Forum and Aerospace Exposition, Nashville, TN, Jan. 2012.

[6] Woodward, P., and Colella, P., "The Numerical Simulation of Two-Dimensional Fluid Flow with Strong Shocks," Journal of Computational Physics, Vol. 54, 1984, pp. 115173.

[7] Figueira da Silva, L. F., Azevedo, J. L. F., and Korzenowski, H., "Unstructured Adaptive Grid Flow Simulations of Inert and Reactive Gas Mixtures," Journal of Computational Physics, Vol. 160, No. 2, May 2000, pp. 522-540.

[8] Roe, P. L., "Approximatte Riemann Solvers, Parameter Vectors, and Difference Schemes," Journal of Computational Physics, Vol. 43, No. 2, 1981, pp. 357-372.

[9] Shu, W. C., "TVB Uniformly High-Order Schemes for Conservation Laws,” Mathematics of Computation, Vol. 49, 1987, pp. 105-121.

[10] Wang, Z. J., "Spectral (Finite) Volume Method for Conservation Laws on Unstructured Grids: Basic Formulation," Journal of Computational Physics, Vol. 178, No. 1, May 2002, pp. 210-251.

[11] Wang, Z. J., and Liu, Y., "Spectral (Finite) Volume Method for Conservation Laws on Unstructured Grids II: Extension to Two-Dimensional Scalar Equation," Journal of Computational Physics, Vol. 179, No. 2, Jul. 2002, pp. 665-698.

[12] Knuth, D. E., The Art of Computer Programming. 3: Sorting and Searching (2nd ed.), Addison-Wesley, Reading, MA, 1998. 
[13] Liu, Y., and Vinokur, M., "Exact Integrations of Polynomials and Symmetric Quadrature Formulas over Arbitrary Polyhedral Grids," Journal of Computational Physics, Vol. 140, No. 1, Feb. 1998, pp. 122-147.

[14] van den Abeele, K., and Lacor, C., "An Accuracy and Stability Study of the 2D Spectral Volume Method," Journal of Computational Physics, Vol. 226, No. 1, Sept. 2007, pp. 1007-1026.

[15] Gooch, C., "Quasi-ENO Schemes for Unstructured Meshes Based on Unlimited DataDependent Least-Squares Reconstruction,' Journal of Computational Physics, Vol. 133, 1997, pp. 6-17.

[16] Friedrich, O., "Weighted Essentially Non-Oscillatory Schemes for the Interpolation of Mean Values on Unstructured Grids," Journal of Computational Physics, Vol. 144, No. 1, July 1998, pp. 194-212.

[17] Jiang, G. S., and Shu, C. W., "Efficient Implementation of Weighted ENO Schemes," Journal of Computational Physics, Vol. 126, No. 1, June 1996, pp. 77-99.

[18] Abgrall, R., "On Essentially Non-oscillatory Schemes on Unstructured Meshes: Analysis and Implementation," Journal of Computational Physics, Vol. 114, 1994, pp. 45-58.

[19] Harten, A., and Chakravarthy, S. R., "Multi-Dimensional ENO Schemes for General Geometries,” ICASE Report 91-76, 1991.

[20] Emery, A., "An Evaluation of Several Differencing Methods for Inviscid Fluid Flow Problems," Journal of Computational Physics, Vol. 2, 1968, pp. 306-331.

[21] Sonar, T., "On the Construction of Essentially Non-Oscillatory Finite Volume Approximations to Hyperbolic Conservation Laws on General Triangulations: Polynomial Recovery, Accuracy and Stencil Selection," Comput. Methods Appl. Mech. Engr., Vol. 140, No. 2, 1997, pp. 157-181. 